\title{
Atypical femoral fractures associated with long-term bisphosphonate use
}

\author{
Soumya Chatterjee MD MS
}

Competing interests: None declared.

This article has been peer reviewed.

Correspondence to: Soumya Chatterjee, chattes@ccf.org

CMAJ 2013. DOI:10.1503 /cmaj.121698

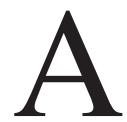
75-year-old woman with osteoporosis presented with a 2-year history of anterior thigh pain that was worse when standing and walking. She had taken alendronate for more than 20 years, which was replaced with annual zoledronic acid infusions for the last 3 years. On examination, she had an antalgic gait. The range of motion of her hips, knees and straight leg raise were normal. The results of a complete blood count and metabolic panel were normal, as were her levels of vitamin D, muscle enzymes and acute phase reactants. Her thyroid function was normal.

Radiographs showed cortical thickening along the lateral aspects of the midfemoral shafts of both legs, with associated horizontal lucency within the thickened lateral cortex, consistent with stress fractures (Figure 1). Bone scintigraphy showed abnormal foci of increased tracer uptake corresponding to the nondisplaced frac-

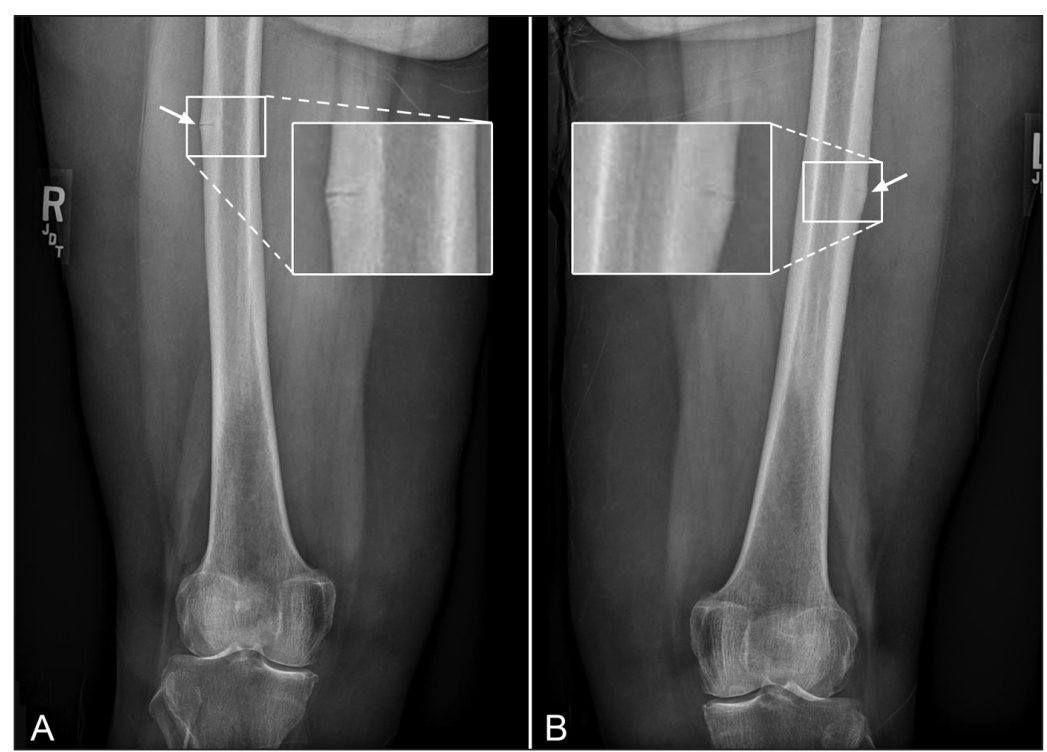

Figure 1: Radiographs showing cortical thickening along the lateral aspects of the right (A) and left (B) femoral shafts with associated horizontal lucency within the thickened lateral cortex, consistent with stress fractures (arrows) in a 75-year-old woman with osteoporosis. Magnified views are shown in the insets to show the horizontal lucencies. tures on the radiographs. She was advised to avoid bisphosphonates and start daily subcutaneous injections of teriparatide (recombinant parathyroid hormone). She underwent prophylactic fixation of both femurs with intramedullary nails.

Long-term use of bisphosphonates (especially alendronate) has been associated with subtrochanteric femoral cortical stress reaction. ${ }^{1}$ The risk of atypical subtrochanteric fractures increases with the duration of bisphosphonate exposure. ${ }^{1}$ Often there is prodromal pain for weeks to years..$^{1,2}$ In a case-control study, the incidence rate was quite low (32 cases per million person-years). ${ }^{1}$ Other risk factors included age between 50 and 79 years, female sex and the use of vitamin D and glucocorticoids. ${ }^{1}$ Bilateral fractures were frequent.

A transverse fracture line through the hypertrophied lateral cortex of the femoral shaft is characteristic on radiographs. ${ }^{1}$ The proposed mechanism is prolonged suppression of normal bone remodeling, with impaired repair of microfractures and increased bone fragility. ${ }^{1}$ Atrophic nonunion is common.

Although the risk of atypical subtrochanteric fracture seems to rapidly diminish after stopping bisphosphonates, ${ }^{2}$ the recommendation to discontinue use in patients with osteoporosis is controversial. Patients should be informed that the benefit of bisphosphonates appears to diminish after 5 years of continuous use, ${ }^{3}$ and they should be cautioned about the increased risk of this potential complication.

\section{References}

1. Meier RP, Perneger TV, Stern R, et al. Increasing occurrence of atypical femoral fractures associated with bisphosphonate use. Arch Intern Med 2012;172:930-6.

2. Bauer DC. Atypical femoral fracture risk in patients treated with bisphosphonates. Arch Intern Med 2012;172:936-7.

3. Black DM, Schwartz AV, Ensrud KE, et al. Effects of continuing or stopping alendronate after 5 years of treatment: the Fracture Intervention Trial Long-term Extension (FLEX): a randomized trial. JAMA 2006;296:2927-38

Affiliations: Lerner College of Medicine of Case Western Reserve University, Department of Rheumatic and Immunologic Diseases, Cleveland Clinic, Cleveland, Ohio 